DOI: https://doi.org/10.46296/gt.v4i8edesp.0034

\title{
PROPUESTA DE INTERVENCIÓN COGNITIVO-CONDUCTUAL PARA EL TRASTORNO DE ESTRÉS POSTRAUMÁTICO
}

\section{COGNITIVE-BEHAVIORAL INTERVENTION PROPOSAL FOR POST- TRAUMATIC STRESS DISORDER}

\author{
Moreira-Navia Jennifer Roxana ${ }^{1}$ \\ ${ }^{1}$ Estudiante de la Maestría Académica con Trayectoria de Investigación en Psicología, Mención \\ Psicoterapia. Instituto de Posgrado de la Universidad Técnica de Manabí, UTM. Portoviejo, \\ Ecuador. Correo: jmoreira4300@utm.edu.ec. ORCID ID: https://orcid.org/0000-0001-8434-2443
}

\begin{abstract}
Resumen
Se describe el caso de una mujer de 18 años con trastorno de estrés postraumático, desarrollara a partir de una internación hospitalaria por una enfermedad sin diagnosticar. Su sintomatología, los diversos exámenes y la experiencia hospitalaria, en la que presenció varias muertes, se vio acompañada de ansiedad e irritabilidad. Luego de que se interviniera quirúrgicamente y se le diera el alta, continua la ansiedad, acompañado de sintomatología postraumática. Se procede a la revisión del caso mediante entrevistas y aplicación de pruebas psicométricas para la obtención de un diagnóstico y posterior propuesta psicoterapeuta desde el modelo cognitivo-conductual.
\end{abstract}

Palabras clave: psicología, plan, psicoterapia, trauma.

\begin{abstract}
We describe the case of an 18-year-old woman with post-traumatic stress disorder, developed from a hospital stay for an undiagnosed illness. His symptoms, the various examinations and the hospital experience, in which he witnessed several deaths, was accompanied by anxiety and irritability. After he underwent surgery and was discharged, the anxiety continues, accompanied by post-traumatic symptoms. The case is reviewed through interviews and application of psychometric tests to obtain a diagnosis and subsequent psychotherapist proposal from the cognitive-behavioral model.
\end{abstract}

Keywords: psychology, plan, psychotherapy, trauma.

Información del manuscrito:

Fecha de recepción: 13 de julio de 2021.

Fecha de aceptación: 27 de septiembre de 2021.

Fecha de publicación: 12 de noviembre de 2021. 


\section{Introducción}

El trastorno de estrés postraumático se caracteriza por el malestar causado en el individuo a causa de la exposición de un evento traumático o del impacto de algún evento ocurrido a alguien más, como en el caso de muerte de otras personas. El revivir el evento traumático es uno de los aspectos más molestos en este trauma, así como la evitación a lugares que le recuerden el evento traumático. Pueden estar acompañados de pesadillas, cambios del estado de ánimo, hipervigilancia, etc. $\mathrm{El}$ malestar que causa es significativo.

Las técnicas cognitivas conductuales son las que han dado mejores resultados según estudios. Entre las más usadas están las técnicas de relajación, modificación de creencias, desensibilización sistemática y técnica de exposición.

Las técnicas de relajación, en especial las de respiración, son muy eficaces en este tipo de trastornos. Son una base o una forma de preparar el terreno para poder trabajar con el paciente. Según Rodríguez, T., García, C. Y Cruz, R. (2005), las técnicas de relajación son aquellas técnicas que, actuando sobre los estados psíquicos del sujeto, provocan modificaciones del estado 0 del funcionamiento. Constituye una de las primeras maneras de permitir al paciente estabilizarse junto con la técnica de respiración profunda, ya que permite que el paciente pueda tomar conciencia más fácilmente de lo que pasa a su alrededor.

La reestructuración cognitiva $(R C)$, consiste en que el cliente, con la ayuda inicial del terapeuta, identifique y cuestione sus pensamientos desadaptativos, de modo que queden sustituidos por otros más apropiados y se reduzca o elimine así la perturbación emocional y/o conductual causada por los primeros (Bados, A., 2010). Su eficacia en este tipo de trastornos tiene que ver con el hecho de que, luego del trauma, la persona siente una serie de síntomas asociados al recuerdo del evento. Estos recuerdos son vistos como un peligro real o latente. Es necesario cambiar el significado que se les tribuye a estos recuerdos, permitirle a la persona darse cuenta de qué es racional y qué es irracional Este aprendizaje (RodríguezSaavedra \& Cano-Vindel, 1995) citados por 
Dongil (2008) tiene un gran valor adaptativo, pues asocia un estímulo que ha sido etiquetado como peligroso con una respuesta emocional potente que nos activa y nos lleva a evitar dicho estímulo. Dicho de otro modo, la RC permite que la persona pueda generar conceptos adaptados a partir de sus recuerdos. Muchas veces puede darse sentimiento de culpa $u$ odio que pueden trabajarse muy bien con esta técnica.

Otra de las técnicas es la desensibilización sistémica, orientada a disminuir sintomatología conductual de miedo y evitación. Guerra Y Plaza (2009) hablan sobre la eficacia de esta técnica en su trabajo, en el cual citan a Baguena (2001), quien señala que diversos estudios reportan efectos positivos de la aplicación de la técnica en veteranos de guerra (Bowen y Lambert, 1986, citado en Baguena, 2001), víctimas de accidentes automovilísticos (Muse, 1986, citado en Baguena, 2001), y en mujeres víctimas de violación (Frank et al., 1988, citado en Baguena 2001).

Las técnicas de exposición pretenden romper el círculo que mantiene la evitación induciendo al cliente a afrontar las situaciones 0 estímulos internos temidos (Lopez, A., 2011). Esto permite que el paciente pueda enfrentar sus pensamientos traumáticos y poder tener una mejor reacción. Jaycox y Foa (1996) según Báguena, J (2001) sugieren que el individuo aprende que: (a) los estímulos que le recuerdan el trauma no le causan daño, (b) recordar el trauma no implica revivir la amenaza, (c) existe una habituación de la ansiedad mientras que el individuo permanece próximo a los recuerdos temidos, y (d) la experiencia de ansiedad no concluye en la pérdida de control temida.

El objetivo de este trabajo consiste analizar un caso, realizar un diagnóstico diferencial y el consiguiente plan de trabajo. Para ello, se realizarán entrevistas a la paciente y a su entorno familiar cercano, así como la aplicación de test que, junto al análisis de su sintomatología y usando como herramienta diagnóstica el DSM-V y el CIE10, permitirán un diagnóstico acertado y su correspondiente plan de trabajo, que brindará a la paciente una mejor calidad de vida. 


\section{Presentación del caso}

Mujer de 18 años de edad de nacionalidad ecuatoriana, heterosexual, católica, soltera y sin hijos. Actualmente cursa el último año de Bachillerato y vive con sus padres. Su mamá es ama de casa y su padre trabajador público.

Paciente, a quien se referirá posteriormente como L.M., refiere: "Necesito ayuda con las crisis de ansiedad" "Ansiedad: porque me estreso o por lo pensamientos. Me pongo ansiosa, me pongo inquieta"

\section{Historia del problema actual}

Hace aproximadamente un año L.M. debe de ser internada por presentar crisis hipoglucémicas. Debió de ser internada por varios meses en el hospital Verdy Cevallos, donde tuvieron que realizarle varios exámenes hasta lograr obtener su diagnóstico. Luego de los primeros dos meses de su internamiento, inicia con dificultades para dormir, no quería comer, ni bañarse.

Luego fue trasladada a Quito para su operación. Cuando los médicos cirujanos le dijeron que era una operación riesgosa y que podría "quedarse en el quirófano", se intensifican los síntomas que se dieron anteriormente y tuvo que ser medicada psiquiátricamente. Además, antes de la operación, se hinchó considerablemente.

Su operación coincidió con la aparición de su menarquia, que se le había pospuesto en esos dos meses. Luego de la operación tuvo cólicos menstruales que fueron confundidas por la paciente con problemas postoperatorio y entró en crisis. Luego de esta crisis no quería caminar, estaba "bravísima", quería tirarse de la cama, no quería comer ni bañarse.

El 24 de diciembre del 2019 la pasó internada. Poco antes de año nuevo le iban a dar el alta, pero la dejaron internada por más días. Esos días fueron difíciles.

Cuando le dieron el alta, en 23 de enero, en el baño de su casa, tuvo una hemorragia por una de las cicatrices de su operación. Tuvieron que ir al hospital en bus y en el terminal le dieron nauseas, así que tuvieron que ir al baño donde, nuevamente tuvo una fuerte hemorragia "mi hija se vio llena de sangre, era empapada", refiere su madre. En el hospital le dijeron que era la sangre de la operación que necesitaba salir. 
Actualmente le diagnosticaron que aún tiene parte de su enfermedad y podría tener recaídas. No hay aceptación de su enfermedad.

Es dependiente, con una actitud "infantil para su edad" (referencia materna), insegura, posesiva, explosiva. La relación con su padre es un poco distanciada ya que sus personalidades chocan. La relación con su madre es muy buena.

Entre la sintomatología descrita por la paciente actualmente, se encuentra:

- Dificultades para dormir (tarda hasta dos horas).

- Miedo a tener una recaída y estar sola.

- Recuerdo de lo vivido como si lo estuviese viviendo nuevamente.

- Pesadillas en relación a lo que vivió.

- Miedo a morir a causa de su enfermedad.

- Durante las crisis: sudoración, palpitación, aumento del ritmo cardiaco, mareos, sudoración en las manos.
- Se dan en momentos de calma en lugares como la iglesia y en sitios asociados al evento vivido, como hospitales.

- Las crisis a veces se confunden con los síntomas de sus crisis hipoglucémicas. Manifiesta que se le comienzan a "cruzar pensamientos de lo que viví". Se pone nerviosa, con ganas de llorar y siente que no se puede tranquilizar.

- Los pensamientos que se le suelen venir en una crisis es el miedo a morir, miedo a que no haya nadie que pueda ayudarla, miedo a estar pasando por una crisis hipoglucémica.

- Los recuerdos de loe eventos vividos son: "siento como si me estuvieran pinchando", "cuando una noche me pincharon mucho porque no me encontraban las venas", "cuando me sacaron los drenajes", cuando me sacaron sangre por el pie", "cuando me pusieron la vía central".

\section{Antecedentes personales}

L. M fue planeada y deseada. Su madre debió de someterse a un tratamiento para quedar embarazada y sin riesgos, ya que 
antes de ella, sus padres tuvieron una hija con hidrocefalia que murió al poco tiempo de nacida.

Durante su embarazo no tuvo problemas, mal embarazo, sustos ni necesidad de tomar medicamente. Su nacimiento fue normal, lloró al nacer, sin coloración extraña ni alteraciones. Les dieron el alta el mismo día.

Realizó el jardín a los cinco años sin inconvenientes. A los seis nace su hermano menor con hidrocefalia y sus padres tuvieron que estar fuera de casa por mucho tiempo. A penas llegaban a casa por tres días y debían de irse nuevamente porque su hermano se ponía muy mal y podría morir. En ese tiempo vivió con su abuela materna. En su escuela le hacían bullying a causa de su hermano. La cambiaron de escuela y su situación mejoró.

El resto de su primaria no tuvo inconvenientes. A los 12 años fue internada por apendicitis sin inconvenientes. Algo relevante es que vio muchos de los procesos médicos a los que se sometió su hermano quien, a causa de su enfermedad, pasó parte de su vida en hospitales y con médicos.
En secundaria tuvo algunas amistades no muy buenas. Solía apegarse a algunas amigas $y$, aunque se llevaba bien con los demás, no entablaba mayores relaciones con ellos. Solía ser posesiva y aún lo sigue siendo. A eso se suma que su madre debió de ausentarse a causa de la enfermedad de su hermano.

A los 16 años su hermano murió y fue un golpe muy fuerte para ella, pero sentía que debía ser fuerte por los demás. 8 meses después murió su mascota Se mudaron después de eso. Su madre entró en depresión. La muerte de su hermano afectó a todo.

Un años después de la muerte de su hermano, deben internarla por sus crisis hipoglucémicas, una dificultad que también tenía su hermano.

\section{Antecedentes psicopatológicos}

\section{personales}

- Operación por gastritis a los 12 años

- Operación por tumores en el páncreas a los 17 años

\section{Antecedentes familiares}

- Tío materno con depresión diagnosticada 
- Abuela materna con depresión diagnosticada desde hace 10 años a raíz de que le sacaron el útero.

- Madre con depresión mayor a raíz de la muerte de su hijo hace dos años. Medicada psiquiátricamente por casi un año. Actualmente no toma medicación y es resiliente.

\section{Pruebas realizadas}

1. Escala de Síntomas de Estrés Postraumático (PSS): Tes utilizado para medir la presencia o no de dicho estrés. El resultado de la evaluación es de 30 puntos sobre 51 , siendo un puntaje alto vinculado a este trastorno. Adicional revela interferencia en algunas áreas de su vida como la relación con su familia y la satisfacción de la vida en general.

2. Escala de valoración de Hamilton para la ansiedad: El puntaje obtenido fue de 16 , lo que significa que tiene hay la presencia de ansiedad entre leve y moderada.

3. Cuestionario Abreviada del Trastorno de Pánico (CATP): El factor físico tuvo un puntaje de 7 sobre16. El factor mental tuvo un puntaje de 9 sobre 16. El factor social tuvo un puntaje de 1 sobre 16. El total fue de un 17 sobre 48, lo que revela ausencia de ataque de pánico.

\section{Hipótesis diagnóstica}

Luego de la revisión de los síntomas, su historia clínica y las pruebas realizadas, se concluye que la paciente L.M. de 18 años, presenta un cuadro de Trastorno de estrés postraumático 309.81 (F43.10)

\section{Plan terapéutico (Modelo cognitivo conductual)}

Objetivo general: Reducir los síntomas del Trastorno del Estrés postraumático mediante el uso de técnicas cognitivos conductuales.

Tabla 1. Modelo de plan de trabajo.

\begin{tabular}{lllll}
\hline \# & Objetivo específico & $\begin{array}{l}\text { Técnica } \\
\text { utilizar }\end{array}$ & a & Descripción de la técnica \\
\hline $\mathbf{1} \quad \begin{array}{l}\text { Conocer las } \\
\text { demandas iniciales } \\
\text { del paciente y su } \\
\text { anamnesis personal }\end{array}$ & $\begin{array}{l}\text { Entrevista } \\
\text { indual }\end{array}$ & $\begin{array}{l}\text { Entrevista inicial de rutina. Datos de filiación, } \\
\text { como sintomatología. }\end{array}$ \\
& &
\end{tabular}


Ampliar la Entrevista Entrevista complementaria. Consiste en información brinda parental conocer desde el punto de vista de los

2 por la paciente desde padres su caso.

el punto de vista Por la edad de la paciente y al estar en paterno. bachillerato aún, se tomó esta medida.

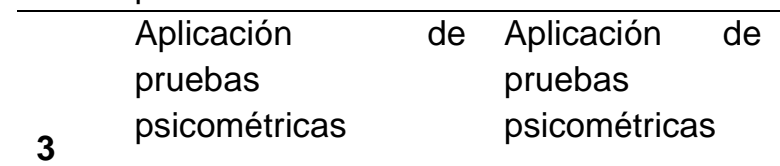

Se explica a la paciente el motivo de los test y su finalidad.

tiempo de inicio y de final.
Se observa reacciones mientras responde las preguntas.

\begin{tabular}{|c|c|c|c|}
\hline 4 & $\begin{array}{l}\text { Brindar conocimiento } \\
\text { sobre sus signos y } \\
\text { síntomas }\end{array}$ & Psicoeducación & $\begin{array}{l}\text { Se da luces a la paciente sobre sus signos y } \\
\text { síntomas. } \\
\text { Se explica su procedencia y el plan } \\
\text { terapéutico a breves rasgos. }\end{array}$ \\
\hline 5 & $\begin{array}{l}\text { Proporcionar una } \\
\text { primera medida para } \\
\text { controlar la aparición } \\
\text { de los síntomas. }\end{array}$ & $\begin{array}{l}\text { Técnica de } \\
\text { relajación: } \\
\text { respiración } \\
\text { diafragmática }\end{array}$ & $\begin{array}{l}\text { Se aplica la técnica de respiración } \\
\text { diafragmática en tiempos de } 4 \text { y } 8 \text { (inhalar y } \\
\text { exhalar). Se explica su objetivo y beneficios. } \\
\text { Se trabajará con búsqueda de lugar seguro, } \\
\text { ya que se va a trabajar con eventos que } \\
\text { generan ansiedad. } \\
\text { Dejar como tarea para la casa identificar las } \\
\text { ideas que aparecen cuando tiene síntomas } \\
\text { semejantes a la hipoglucemia y sus } \\
\text { pesadillas. }\end{array}$ \\
\hline 6 & $\begin{array}{lrr}\text { Conocer } & \text { las } & \text { ideas } \\
\text { que tiene la paciente } \\
\text { sobre el origen de su } \\
\text { trauma y } \\
\text { generan } \\
\text { malestar. }\end{array}$ & $\begin{array}{l}\text { Identificación de } \\
\text { creencias } \\
\text { irracionales } \\
\text { (restructuración } \\
\text { cognitiva) }\end{array}$ & $\begin{array}{l}\text { Revisar las ideas previas asociadas al } \\
\text { trauma que puedan generar malestar. Se } \\
\text { identifican sus ideas, sus origen y el grado } \\
\text { de malestar. }\end{array}$ \\
\hline 7 & $\begin{array}{l}\text { Dar explicaciones } \\
\text { racionales a las ideas } \\
\text { irracionales } \\
\text { descubiertas. }\end{array}$ & $\begin{array}{l}\text { Identificación y } \\
\text { modificación de } \\
\text { creencias } \\
\text { irracionales } \\
\text { (restructuración } \\
\text { cognitiva) }\end{array}$ & $\begin{array}{l}\text { A partir de las ideas irracionales analizadas } \\
\text { anteriormente, se procede a dar un } \\
\text { significado racional a todas ellas o a las que } \\
\text { generan mayor malestar. }\end{array}$ \\
\hline 8 & $\begin{array}{lr}\text { Reducir el } & \text { nivel de } \\
\text { ansiedad } & \text { que } \\
\text { generan } & \text { los } \\
\text { recuerdos del evento }\end{array}$ & $\begin{array}{l}\text { Técnica de } \\
\text { desensibilizació } \\
\mathrm{n} \text { sistematizada } \\
\text { (pensamientos) }\end{array}$ & $\begin{array}{l}\text { Se hace una lista de los recuerdos que suele } \\
\text { tener en los flashbacks. } \\
\text { A continuación, se le pide que se coloque en } \\
\text { una posición cómoda y que se relaje. Se } \\
\text { puede usar las técnicas de relajación. } \\
\text { Luego se le pide que recuerde algún evento } \\
\text { traumático con la mayor claridad. No debe } \\
\text { de ser invasivo ni muy extenso. } \\
\text { Luego de esto, se le pide que verbalice el } \\
\text { nivel de malestar que le genera y se procede } \\
\text { a hacer lo mismo }\end{array}$ \\
\hline 9 & $\begin{array}{lr}\text { Reducir el } & \text { nivel de } \\
\text { ansiedad } & \text { que } \\
\text { generan } & \text { los } \\
\text { recuerdos del evento }\end{array}$ & $\begin{array}{l}\text { Técnica de } \\
\text { desensibilizació } \\
\text { n sistematizada }\end{array}$ & $\begin{array}{l}\text { Se traba con los mismos pensamientos que } \\
\text { se trabajaron en la sesión anterior y se } \\
\text { realiza una comparación en el nivel de }\end{array}$ \\
\hline
\end{tabular}


malestar que generan los pensamientos esta sesión con las anteriores.

\begin{aligned} \hline & Reducir el nivel de & Técnica de \\ ansiedad & que & desensibilizació \\ generan & los & n sistematizada \\ 10 & recuerdos del evento & \end{aligned}

Se muestran al paciente imágenes semejantes a los descritos en sus flashbacks e incluso elementos materiales. Se le pide que los observe y se registran niveles de malestar.

Se podría trabajar usando los recuerdos también.

\begin{tabular}{lrlrl}
\hline & $\begin{array}{l}\text { Reducir el nivel de } \\
\text { ansiedad } \\
\text { generan } \\
\text { recuerdos del evento }\end{array}$ & $\begin{array}{l}\text { Terapia } \\
\text { losposición }\end{array}$ & $\begin{array}{l}\text { de } \\
\text { vivo }\end{array}$ & $\begin{array}{l}\text { Luego de haber realizado el proceso } \\
\text { anterior, se acompaña a la paciente a algún } \\
\text { lugar que le recuerde su evento traumático. } \\
\text { Si es necesario, se podría añadir una sesión } \\
\text { más hasta que finalmente logre ingresar. }\end{array}$ \\
\hline $\begin{array}{llll}\text { Finalizar el proceso } \\
\text { terapéutico y revisar } \\
\text { los aprendizajes. }\end{array}$ & $\begin{array}{l}\text { Revisión } \\
\text { proceso } \\
\text { terapéutico } \\
\text { cierre }\end{array}$ & del & $\begin{array}{l}\text { Se realizará una comparación entre el inicio } \\
\text { del proceso y el final. }\end{array}$ \\
& & ye revisará si se cumplió el objetivo. & $\begin{array}{l}\text { Se recordará los aprendido y se agendarán } \\
\text { las sesiones de seguimiento. } \\
\text { Se realiza el cierre y la despedida. }\end{array}$
\end{tabular}

\section{Conclusiones}

La internación hospitalaria, acompañada de las pruebas exhaustivas para llegar a su diagnóstico y la operación riesgosa a la que fue sometida, es una parte significativa para poder llegar al diagnóstico, y diferenciarlo de otros trastornos semejantes, como las fobias o el trastorno de pánico.

La entrevista con los padres arrojó mucha información que a la paciente se le pasaba por alto y brindaron otro punto de vista distinto a la de L.M., que más adelante sirvió para la identificación del trastorno. Se ratifica la importancia de entrevistar no solo al paciente, sino a quién la acompaña a entrevista o a quién convive con este.

Las técnicas cognitivas conductuales son las más utilizadas en el tratamiento del trastorno de estrés postraumático, demostrando eficacia en la disminución de síntomas, por lo que se espera que, con el modelo del plan terapéutico trazado, la paciente reduzca el malestar presente y mejorar su calidad de vida.

El objetivo del plan terapéutico está destinada a la disminución de los síntomas que generan malestar. Está dividido en doce sesiones de entre 30 a 45 minutos. Se recomienda el seguimiento con la 
familia y el compromiso de esta para mejores resultados.

Cada caso tiene características diferentes, por lo que el objetivo y el plan de trabajo está destinado a cubrir las necesidades de cada paciente. Es por ello que la revisión de la información, una correcta entrevista y diagnóstico, son indispensables para una correcta psicoterapia.

\section{Bibliografía}

Bados, A. (2010). La técnica de la reestructuración cognitiva [Universidad de Barcelona]. http://diposit.ub.edu/dspace/bi tstream/2445/12302/1/Reestr ucturaci\%C3\%B3n.pdf

Báguena, J (2001). Tratamientos psicológicos eficaces para el estrés post-traumático. Psicothema, 13 (3), 479-492. https://www.redalyc.org/pdf/7 27/72713313.pdf

Dongil, E. (2008). Reestructuración cognitiva: un caso de estrés postraumático. Ansiedad $\mathrm{y}$ Estrés, 14(2) 265-288. https://webs.ucm.es/info/seas /ta/trat/Tratamiento_cognitivoconductual_caso_trastorno_e stres_postraumatico_violacio n.pdf

Guerra Y Plaza (2009). Tratamiento Cognitivo-Conductual del Estrés Postraumático en un caso de violación infantil. Revista de Psicología, 18(1). file://C:/Users/ASUS/Downlo ads/17130-1-50141-1-1020111106.pdf

López, A. (2011). Técnicas de exposición [Universidad de Barcelona].

http://diposit.ub.edu/dspace/bi tstream/2445/18403/1/T\%C3 $\%$ A9cnicas\%20de\%20Exposi ci\%C3\%B3n\%202011.pdf

Rodríguez, T., García, C. y Cruz, R. (2005). Técnicas de relajación y autocontrol emocional. MediSur 3 (3), 5570.

https://www.redalyc.org/pdf/1 800/180019787003.pdf 